\title{
What can we learn from high energy flares in the Fermi sample of FSRQs: from a case study to dozens of objects
}

\author{
Luigi Pacciani ${ }^{1,3, a}$, Fabrizio Tavecchio ${ }^{2}$, Immacolata Donnarumma ${ }^{1,3}$, and Antonio Stamerra ${ }^{4}$ \\ ${ }^{1}$ INAF-Istituto di Astrofisica e Planetologia Spaziale, Via Fosso del Cavaliere 100, I-00133 Rome, Italy \\ ${ }^{2}$ INAF-Oservatorio Astronomico di Brera, via E. Bianchi 46, I-23807, Merate, Italy \\ ${ }^{3}$ INFN sez. Tor Vergata, Via della Ricerca Scientifica 1, I-00133 Rome, Italy \\ ${ }^{4}$ INAF-Osservatorio Astrofisico di Torino, via P. Giuria 1, I-10125 Torino, Italy
}

\begin{abstract}
In 2008 the flat spectrum radio quasar GB6 J1239+0443 ( $\mathrm{z}=1.762$ ), underwent an high activity period at all wavelengths. In particular, at optical-uv wavelengths, the source underwent a flux enhancement of a factor 15-30 in 6 years, and $>10$ in half a year, showing a transition from accretion disk to synchrotron jet dominated emission. During the flare, Fermi observed a flat gamma-ray spectrum, extended up to $15 \mathrm{GeV}$, with no statistically-significant absorption from the broad line region (BLR), suggesting that the blazar-zone is located beyond it. We obtained the same findings from the modeling of the broad-band spectral energy distribution for the flaring activity periods, which is well constrained by the multiwavelength data collected, and from the accretion disk luminosity and black hole mass that we estimated from the archival data. Other authors suggested that 4C +21.35 , PMN J2345-1555, and 3C 279 underwent high energy flares originating from outside or just beyond the BLR. We report here the first results obtained in our study, while we will report the results on the complete sample in a forthcoming paper where we will show the multiwavelength spectra on a dozen of FSRQ which we propose radiate gamma-rays far from the central SMBH, and we will discuss the scenarios originating from this result.
\end{abstract}

\section{Introduction}

Blazars are Active Galactic Nuclei, with jet orientation close to the line of sight of the observer [1]. These objects emit from radio band to gamma-ray. Fifty of these objects have been detected in the $\mathrm{TeV}$ range [2]). Blazars show high gamma-ray activity and variability above $100 \mathrm{MeV}$. The source of the emission is believed to be the nonthermal emission from the relativistic jet, overwhelming the thermal components, but the emission mechanisms are still debated. Hadronic and leptonic models were developed to explain the observations. In the framework of leptonic models, the gamma-ray emission is explained with the Inverse-Compton mechanism. Two scenarios are invoked : the Synchrotron Self Compton (SSC) of the relativistic electrons from the jet with the photon field coming from the synchrotron radiation produced by the same relativistic electron population itself $[3,4]$, which is usually invoked for the BL Lac objects. The high energy emission of Flat Spectrum Radio Quasars (FSRQs) is usually modeled in the framework of External Compton (EC) scenario, which foresees an external photon field as the seed for the inverse-Compton process by the relativistic electrons. The external photon field can be identified with the thermal radiation from the disk at least reprocessed by the Broad Line Regions (BLR) or by the

a. e-mail: luigi.pacciani@iaps.inaf.it jet itself, or with the thermal radiation from an hot corona, or from the dusty torus [5]. Depending on the distance of the emitting region from the central Black Hole, the electrons interaction with photon seeds from one region prevails on the others.

Ghisellini and Tavecchio [7] proposed a parametrization of the seed photons intensity as a function of the disk luminosity, and of the location of the emitting region (the blazar-zone).

Poutanen and Stern [8] evaluated that the dense environment of the BLR causes absorption features in the gamma-ray spectrum at least for the FSRQs with the most powerful accretion disk emission $\left(\mathrm{L}_{\text {disk }} \sim 10^{45}-10^{46} \mathrm{erg} \mathrm{s}\right.$ $-1)$.

There are a few sources for which authors claim that the SEDs modeling require far dissipation region at the edge, or outside the BLR : $4 \mathrm{C}+21.35$ (dissipation region $>0.1$ pc $[9,10]$ ), PMN J2345-1555 (dissipation region = $0.1 \mathrm{pc}[11]$ ), 3C 279 (dissipation region $=0.07-0.26 \mathrm{pc}$ $[12,13]$ ), PKS 1510-089 (dissipation region $=0.07-3.2$ pc [14]).

Other authors correlated the time-dependent polarimetric radio images at 43 and $86 \mathrm{GHz}$, the optical polarimetry, and radio, optical, X-, gamma-ray light curves, and claimed that the low and high energy emission is located near the $43 \mathrm{GHz}$ core, at a distance of the order of tens 
of parsecs from the $\mathrm{BH}$ for 3C 454.3 [15]. A similar investigation, performed for the BL Lac objects OJ287 and AO $0235+164[16,17]$, led to similar results.

Fast variability with respect to the veriability inferred from typical size of emitting-regions located at long distances from the supermassie black hole (SMBH) has become a common observative fact in TeV Blazars.

Several theoretical models could account for fast variability of flares occurring at long distances form the central black hole. Among that, Bromberg and Levinson [18] propose that radiative cooling of the shocked outflow layer can lead to focusing and reconfinement of the outflow, explaining the rapid variability at large distances from the SMBH as observed in several TeV blazars.

Magnetic reconnection mechanism could account for rapid variability : Giannios [19] have recently elaborated a minijet model for flares of blazars. He argued that in blazar-jets, a large number of plasmoids could originate from the fragmentation of the reconnection layer. The emission of these plasmoids overlaps, so that what is observed is an envelope of emission from the plasmoids, with time scales of a day. Occasionally a plasmoid could grow and give rise to a fast flare.

\section{Our sample of High Energy FSRQs}

In the second FERMI-LAT catalog [6] gamma-ray spectra are reported integrated for 24 months. The majority of the gamma-ray spectra of FSRQ reported in that catalog have photon indices $>2$, or steeper.

If we restrict the integration times to flaring activity periods, we obtained from the data in the FERMI-LAT archive that there is an handful of objects that show an almost flat gamma-ray spectrum during flares.

These objects do not show the absorption features proposed by Poutanen and Stern [8] due to the Broad Line Region photons, or weak absorption features appear.

For these objects we can infer that in a standard BLR geometry, the emission originates from outside the BLRs. In this scenario, the parametrization proposed by Ghisellini and Tavecchio [7] for the gamma-ray emission allows for the location of the blazar-zone. It is worth mentioning that the Ghisellini and Tavecchio parametrization foresees a steep variation of the gamma-ray emission, varying the location of the emission region. Reversing the problem, in the Spectral Energy Density (SED) modeling, the location of the blazar zone slowly varies with the gamma-ray emission, and thence with the disk luminosity. Thereby, the location of the blazar zone is a stable solution of the SED modeling, which does not vary dramatically changing the disk luminosity input parameter.

With these considerations, we started a deep search in the FERMI-LAT data archive with the aim of locating the blazar zone for this small sample, to try to quantify the relevance of the sample with respect with the whole FSRQs FERMI-LAT catalog, and to match the observative facts with the existing models. At the same time we started to trigger Swift ToO at the occurrence of new High Energy flares, detected on-time from the continuous analysis of incoming FERMI-LAT data.

We report here the first two sources we found. The first one, GB6 J1239+0443 found in the archival FERMI-LAT data, and the second one, PKS B1424-418, found from on-time analysis of fresh data. From this alert we triggered a ToO observation to Swift.

\subsection{GB6 J1239+0443}

GB6 J1239+0443 ( $\mathrm{z}=1.762)$ exhibited two flaring periods that were monitored from optical to gamma-rays. For the first period we have simultaneous observations of INTEGRAL and AGILE on December 2007 - January 2008. INTEGRAL detected the source in optical, and an upper limit only was obtained at hard X-rays. AGILE detected the source for two weeks. The second multiwavelength campaign was performed in January 2009, it involved FERMI-LAT, Kanata, Swift. A detailed description of the two campaigns and of the archival data can be found in [20]. The SEDs obtained with the data of the two campaigns is reported in figure 1 , together with archival optical data.

We were able to evaluate the accretion-disk luminosity from optical archival data obtained during periods of low jet activity, as witnessed by the low radio emission detected simultaneously to the optical observations. We obtained an accretion disk luminosity $\sim 8.9 \times 10^{45}$ erg $\mathrm{s}^{-1}$. The flare of the January 2010 showed a flat gamma-ray spectrum with a photon index $2.14 \pm 0.08$ up to $15 \mathrm{GeV}$, while the photon index reported in the second FERMI-LAT catalog is softer : 2.32. The flat gamma-ray spectrum, with emission up to $20 \mathrm{GeV}$, does not show the adsorption features from gamma-gamma interactions with the Broad Lines, allowing us to assume that the dissipation region is outside or at least at the edge of the BLR.

In this scenario, the high-energy drop of the external Compton could be dominated by the external Compton with photon seeds from the BLR, or from the torus. Thence, from the ratio of the drop of the synchrotron emission to the External Compton emission (note that the high energy drop in External Compton is a lower limit only), we could deduce two upper limit to the magnetic field : a) $<7 \times 10-1$ for the case in which the drop of the External Compton is dominated by the interactions with the BLR seed photons, b) $<7 \times 10-2$ for the case in which the drop of the External Compton is dominated by the interactions with the torus seed photons.

We modeled the SED adopting the Ghisellini and Tavecchio [7] parametrization of the external fields and with the disk luminosity evaluated form the archival nir, optical and uv data. We obtained that the dissipation region is located or at $\sim 0.2 \mathrm{pc}$, or at $\sim 5-7 \mathrm{pc}$ from the 


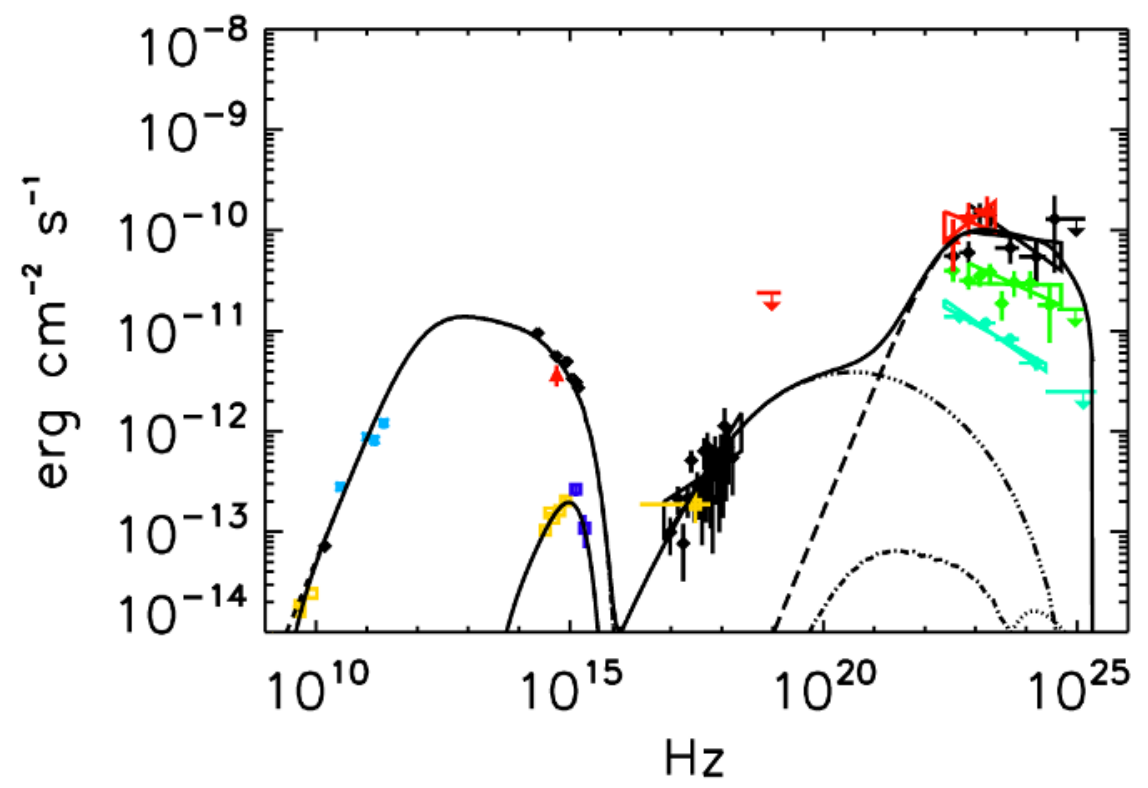

Figure 1. Broad band SEDs of GB6 J1239+0443 for the two multiwavelength campaigns (red for the December 2007-January 2008 campaign, black for the January 2009 campaign), The green gamma-ray spectrum refers to one-month integration of the FERMI-LAT data around the flare of the second campaign. Cyan gamma-ray spectrum refers to the spectrum reported in the second FERMI-LAT catalog. Light blue symbols refer to radio data from PLANCK. The other symbols at nir, optical, uv wavelengths refer to the Sloan Digital Sky Survey archive, and to GALEX. Yellow radio and X-ray symbols refer to archival radio and Rosat all Sky Survey data.

central Black Hole.

The two-fold solution is due to the fact that we could still assume that the interaction of the jet electrons with the BLR seed photons could be responsible for the high energy emission, with the emission region almost loacted at the edge of the BLR. The solution for the model with the far dissipation region is reported in figure 1.

What is interesting is that the solution we found at larger distance could not account for the variability timescales (of the order of a few days) of the flaring activity. This fact could resemble the recollimation scenario [18], but to reconcile the variability timescale with the distant emission we must assume a proton- to electron-number ratio of the order of $10^{-1}$ in the jet.

\subsection{PKS B1424-418}

PKS B1424-418 ( $\mathrm{z}=1.52)$ showed high energy (above $10 \mathrm{GeV}$ ) activity for several months in 2013 . We triggered two ToO observations with Swift in april and may 2013, with the aim of constraining the optical-nuv drop of the synchrotron emission, in flare. We obtained a flat gamma-ray spectrum extended up to $20 \mathrm{GeV}$, as shown in figure 3. The gamma-ray spectrum does not show the absorption features proposed by Poutanen and Stern [8]. Therefore the emitting region must be located outside the BLR.
We found simultaneous nir-optical data thanks to the SMARTS monitoring program of blazars. The SED built for the source during flare gave us two interesting elements : a very low Compton dominance, and a very low magnetic field of $\sim 6 \times 10^{-3} \mathrm{G}$. We evaluated the disk emission starting from the broad $\mathrm{Mg}$ II line luminosity [21], and we obtained a disk luminosity of $10^{46} \mathrm{erg} \mathrm{s}^{-1}$. With these considerations, the SED modeling allowed for one solution only with the blazar-zone at $7 \mathrm{pc}$ from the SMBH. Details of the multiwavelength campaign, of the gamma-ray light curve, and of the SED modeling can be found in [22].

An intriguing consideration is that the gamma-ray light curve reported in figure 2 is similar to the description of the light curve from reconnection events for blazars [19], with an envelop, and several flares. Anyway the timescales are different : for PKS B1424-418 the envelope emission time-scale is of the order of a month and the flaring time-scale is of the order of one day in our case. We note that in the scenario we depicted, the emission region is far away from the $\mathrm{SMBH}$, and the magnetic field is extremely low. Therefore we are in the case of low magnetized plasma, with possibly longer timescales respect to the scenario proposed in [19]. 

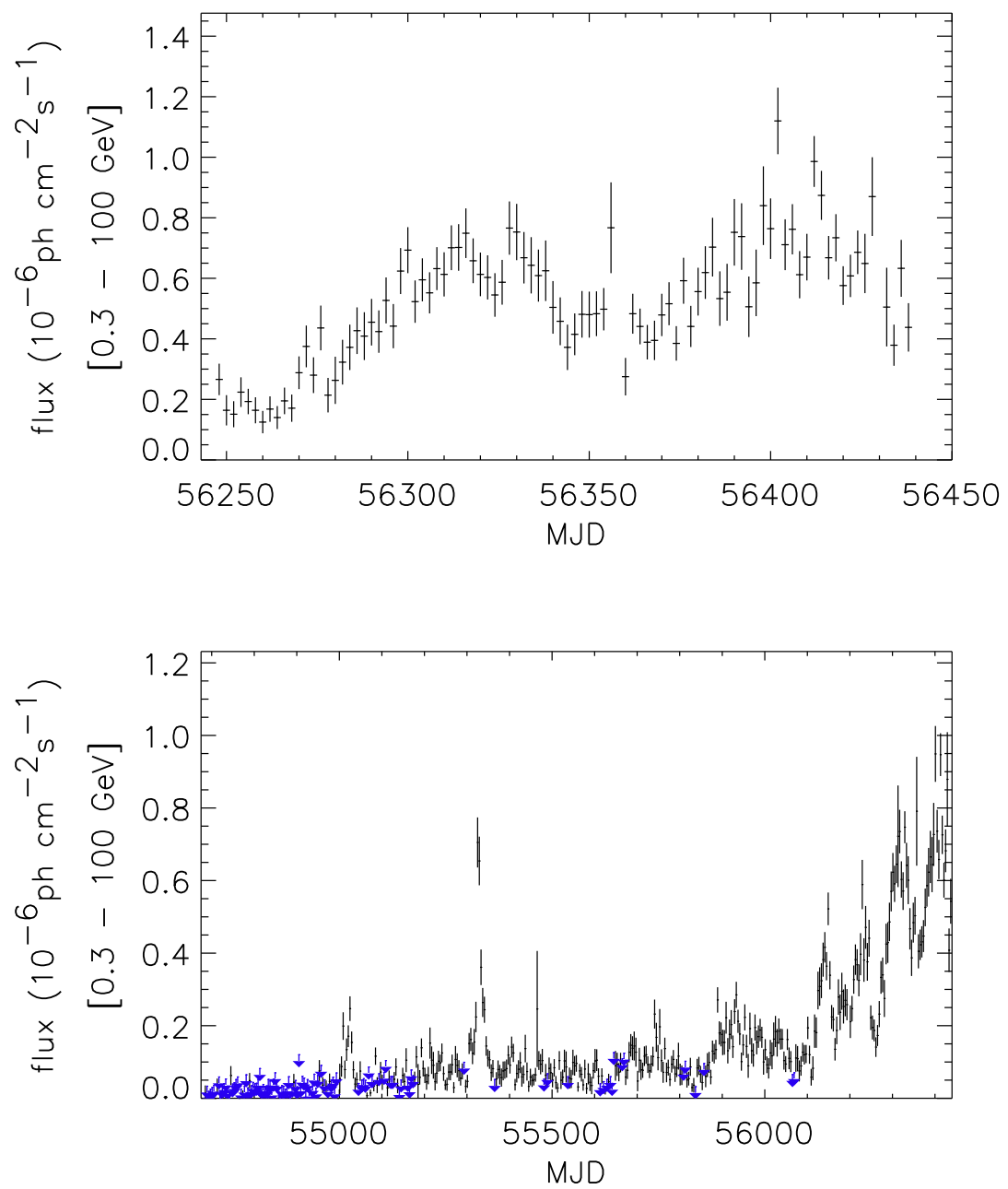

Figure 2. Light curve for PKS B1424-418 in gamma rays. Top : with a binsize of 2 days, Bottom : 4 days.

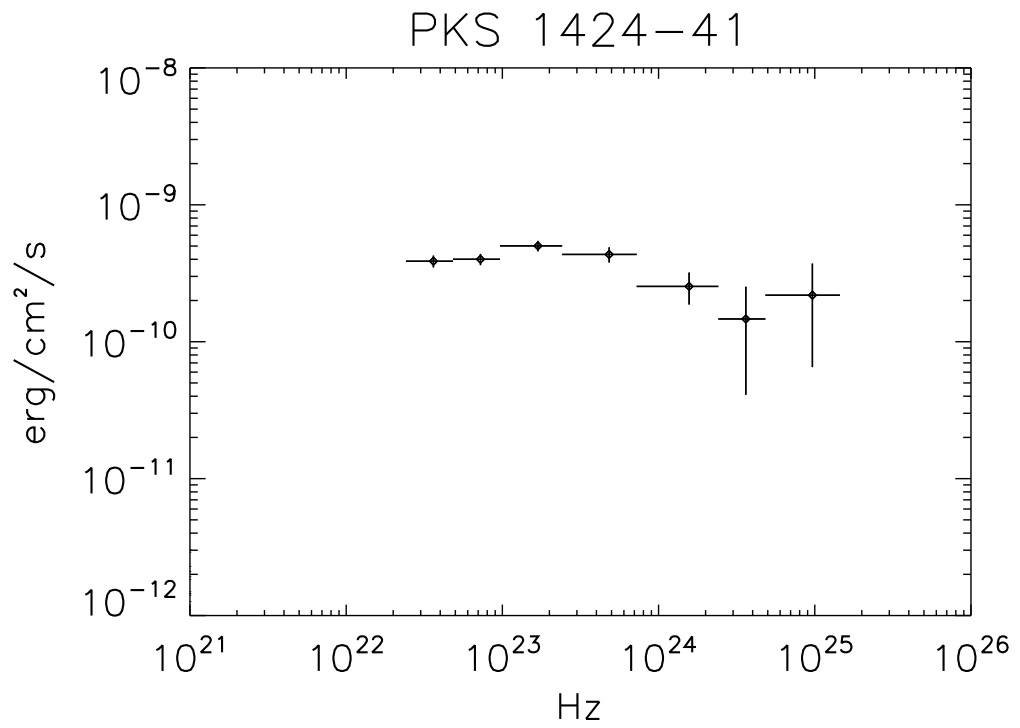

Figure 3. The gamma-ray spectrum of PKS B1424-418 integrated for 4 days around the peak emission on 2013 may $1^{s t}$. 


\section{Considerations and Future plans}

A first result of our study, that we are attempting to confirm, comes from the gamma-ray spectrum of FSRQs during flares : a relatively large fraction of FSRQs have shown flares with blazar-zone outside the BLRs. This result is model independent, and it assume the existence of a BLR surrounding the SMBH.

Concerning the modeling, Tavecchio and Ghisellini [23] have studied various shapes of the BLR, and claim that the photon filed intensity of the BLR does not vary soo much changing the shape of the BLR.

We have shown with the two source reported here, that this study will probe both the reconnection, and the recollimation scenario, in a different environment rather than the one for which these models originated. We have analysed in detail all the FERMI-LAT data archive, searching for high energy flares. For the high energy flares, we searched for multiwavelength simultaneous data, and for archival optical spectra of the flaring sources to derive the disk luminosity from the broad line luminosity.

We plan to enlarge the sample, submitting proposals to Swift, MAGIC for ToO observations triggered with these high-energy flares of FSRQs. A larger sample will allow to quantify the fraction of FSRQ flares emitted far from the SMBH.

\section{References}

[1] Urry, C. M. and Padovani, P., ASP 107 (1995) p. 803845 ;

[2] Albert, J. et al., Science, 320 (2008) p. 1752;

[3] Maraschi, L., Ghisellini, G., and Celotti, A., ApJ, 397 (1992) L5 ;

[4] Marscher, A. P., and Bloom, S. D., 1992, Proceedings of The Compton Observatory Science Workshop, p. 346 ;

[5] Sikora, M., Begelman, M. C., and Rees, M., ApJ, 421 (1994), p. 153;

[6] Nolan, P. L. et al., ApJS 199, 31 (2012)

[7] Ghisellini, G. and Tavecchio, F., MNRAS 397, 985 (2009)

[8] Poutanen, J and Stern, B, ApJL 717, 118 (2010)

[9] Aleksic, J. et al., ApJL 730, 8 (2011)

[10] Tavecchio, F. et al., A\&A 534, 86 (2011)

[11] Ghisellini, G. et al., MNRAS 432, 66 (2013)

[12] Abdo, A. A. et al., Nature 463, 919 (2010)

[13] Hayashida, M. et al., ApJ 754, 114 (2012)

[14] Nalewajko K. et al., ApJ 760, 69 (2012)

[15] Jorstad S. G. et al., ApJ 715, 362 (2010)

[16] Agudo I. et al., ApJL, 726, 13 (2011)

[17] Agudo I. et al., ApJL, 735, 10 (2011)

[18] Bromberg, O. and Levinson A., ApJ 699, 1274 (2009)

[19] Giannios, D., MNRAS 431, 355 (2013)

[20] L. Pacciani et al., MNRAS 425, 2015 (2012)

[21] Stickel, M. et al., A\&AS 80, 103 (1989)

[22] Tavecchio, F. et al., MNRAS 435, 24 (2013)

[23] Tavecchio, F.and Ghisellini, G., MNRAS sumbmitted (arXiv 2012arXiv1209.2291T) 